\title{
Research on the Prediction Model of Total Agricultural Output Value based on Wavelet Neural Network
}

\author{
Liu Jin-yue \\ Computer and Information Technology College \\ Northeast Petroleum University \\ Daqing, Heilongjiang Province, China \\ liujinyue2001@163.com
}

\author{
Zhu Bao-ling \\ Natural Gas Surface Engineering Project Management \\ Department \\ Jilin Oilfield Company \\ Songyuan, Jilin Province, China \\ zhuobaoling@126.com
}

\begin{abstract}
This paper proposes a prediction model of total agricultural output value based on the wavelet neural network, and presents the model structure of network and learning algorithm to train network using the gradient-descent algorithm with the momentum, and then conducts a prediction for our country's total agricultural output value combined with the actual data. To verify the validity of the model, a comparison test was carried out. The results showed that compared with traditional BP network prediction model, this model had a faster convergence rate and higher prediction accuracy.
\end{abstract}

Keywords- wavelet neural network; prediction; total agricultural output value

\section{INTRODUCTION}

As China is a traditional agricultural country, agriculture has been the pillar industry since ancient times, so it is the national economic basis and plays an important role in the successful development of China's economy. Only when the agricultural yield is stable and can meet public requirements, society's long-term stability and sustainable development can be guaranteed. However, agricultural output is a complex non-linear system affected by many factors and with various uncertainties, so the prediction is very difficult.

The artificial neural network has a strong ability to handle complex nonlinear systems, so this theory has been commonly used in the predictive research and obtained satisfactory results. However, there are complex factors affecting agricultural production, if all the factors are regarded as the input variables of neural network without selections, regardless of whether some of these factors have relatively large influences on the agricultural production, but some have little effect which will lead that the input of neural network may contain too much redundant information, thereby leading to the poor generalization ability of the network and inaccurate prediction [1]. According to the literature [1], the rough set theory is adopted to find the main factors affecting agricultural production and utilize the wavelet neural network to carry out prediction for agricultural output value. The wavelet neural network is a kind of feedforward neural network combined with wavelet analysis and neural network theory, which integrates the good time-frequency localization nature of wavelet transform as well as the self-learning function of neutral network. So it has strong approximation and fault-tolerant capacity.
Compared with the forward neural network, the wavelet neural network has the following advantages: first, the primitive of wavelet neural network and the entire structure are determined based on the wavelet analysis theory, which can avoid the blindness on structure design, such as the BP neural network [2]; Second, the wavelet neural network has a stronger learning ability and a faster convergence speed.

By the simulation study of the agricultural output value in the recent thirty years, this paper proposes regarding the major factors affecting agricultural production as the wavelet neural network input and model for the agricultural output value. Its effectiveness and feasibility have been validated.

\section{WAVELET NEURAL NETWORK MODEL}

\section{A. Wavelet Theory}

Wavelet analysis develops from the Fourier transform, aiming at its deficiency. The Fourier transform is the most widely used analytical tool in the field of signal processing, but it has a serious shortage of abandoning the time information in transformation, which can only process stationary signal with unchanged spectral components. However, it would make a huge error in dealing with nonstationary signals, or even the results may be very different from the actual situation. The wavelet transform is the timefrequency analysis method of a signal, which overcomes the defects of short-time Fourier transform in the single resolution and has good localization properties in time domain and frequency domain. Besides, it can achieve the adaptive change of time-frequency window, which is an ideal mathematical tool for local signal spectrum analysis [3].

Wavelet analysis is mainly applied to using a particular method to construct the wavelet base function in specific function space and to expand and approach given signals. The relevant concepts are briefly recounted as follows:

Definition 1: Set $\psi(t) \in L^{2}(R)$, if

$$
\int_{-\infty}^{+\infty} \psi(t) d t=0
$$

and then $\psi(t)$ is regarded as a wavelet, also called the mother wavelet or basic wavelet.

Definition 2: Scale and translate the wavelet function $\psi(t)$, and a family of wavelet functions can be obtained [4]. 


$$
\psi_{a, b}(t)=\frac{1}{\sqrt{a}} \psi\left(\frac{t-b}{a}\right) \quad a \succ 0, b \in R
$$

$\psi_{\mathrm{a}, \mathrm{b}}(t)$ is the wavelet base function of the wavelet $\psi(t)$. In the formula, $a$ is the scale parameter, and $b$ is the translation parameter.

Wavelet transform refers to translating a basic wavelet function $\psi(t)$ by $b$ and then making it form the inner product with the signal $x(t)$ to be analyzed in different scale $a$ [5]. The expression (3) is as follows.

$$
f_{x}(a, b)=\frac{1}{\sqrt{a}} \int_{-\infty}^{\infty} x(t) \psi\left(\frac{t-b}{a}\right) d t \quad a \succ 0
$$

It can be seen from the formula that the wavelet transform is a time-frequency analysis method with variable resolutions. It is able to make adaptive adjustment to sampling step of different frequencies in the time domain. When analyzing low-frequency signals, its time window is large. However, when analyzing high-frequency signals, its time window is small. This conforms to the natural law that the high-frequency signals are with short duration, while the low-frequency signals are with long duration in practical problems. Therefore, as a mathematical theory and analysis method, this method has caused widespread concern.

\section{B. Structure and learning algorithm of wavelet neural network}

Wavelet neural network is based on the BP neural network topology and regarding the wavelet base function as the transfer function of hidden-layer nodes, where the forward propagation occurs in signal and back-propagation occurs in error at the same time [6]. Its topology is shown in figure 1 .

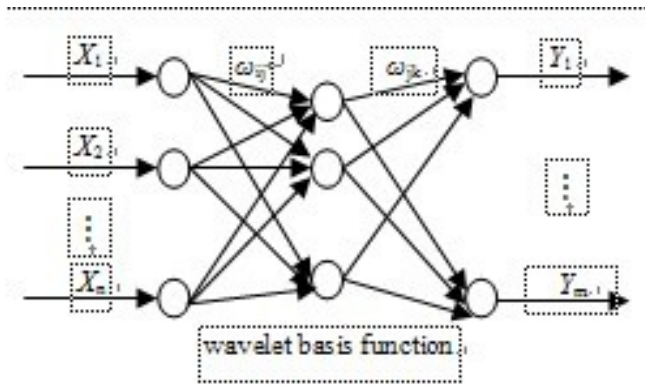

Figure 1. the topological structure of wavelet neural network

In figure. $1, X_{1}, X_{2}, \cdots, X_{\mathrm{n}}$ are the input parameters of wavelet neural network, $Y_{1}, Y_{2}, \cdots, Y_{\mathrm{m}}$ are the predictive output of the network, $\omega_{\mathrm{ij}}$ and $\omega_{\mathrm{jk}}$ are weights of wavelet neural network. When the input signal sequence is $x_{\mathrm{i}}(i=1$, $2, \cdots, k)$, the computational formula (4) of hidden-layer output is shown as follows.

$$
h(j)=h_{j}\left(\frac{\sum_{i=1}^{k} \omega_{i j} x_{i}-b_{j}}{a_{j}}\right) \quad j=1,2, \cdots, l
$$

In this formula, $h(j)$ is the output value of $j^{\text {th }}$ node of the hidden layer; $\omega_{\mathrm{ij}}$ is the connection weight of the input layer and hidden layer; $b_{\mathrm{j}}$ is the shift factor of wavelet basis function; $a_{\mathrm{j}}$ is the expanding factor of the wavelet basis function $h_{\mathrm{j}} ; h_{\mathrm{j}}$ is the wavelet basis function. The wavelet basis function adopted in this paper is Morlet wavelet, and its expression (5) is shown as follows.

$$
y=\cos (1.75 x) e^{-x^{2} / 2}
$$

The calculation formula (6) of the output layer of wavelet neural network is shown as follows.

$$
y(k)=\sum_{i=1}^{l} \omega_{j k} h(i) \quad k=1,2, \cdots, m
$$

In this formula, $\omega_{\mathrm{jk}}$ is the connection weight of hiddenlayer and output layer; $h(i)$ is the output of the ith hiddenlayer node; $l$ is the number of the hidden-layer nodes; $m$ is the number of the output layer nodes.

The training steps of wavelet neural network algorithm are shown as follows:

Step1: network initialization. Randomly initialize the expanding factor $a_{\mathrm{j}}$, shift factor $b_{\mathrm{j}}$, as well as the connection weights $\omega_{\mathrm{ij}}$ and $\omega_{\mathrm{jk}}$ of wavelet function. Set the network learning rate $\eta$;

Step2: classify samples. Samples are divided into training samples and test samples. The training samples are used to train network and the test samples are used to test the accuracy of network prediction.

Step3: predict output. Training samples are input to the network to calculate the output of network prediction and calculate the error $e$ between the network output and desired output.

Step4: correct weight. Correct the network weights and wavelet function parameters according to the error $e$ to make the network predicted values approaching the expectation.

Step5: determine whether the algorithm is over; if it is not, return to step3.

\section{PREDICTIVE MODEL OF AGRICULTURAL OUTPUT}

\section{A. Data preprocessing}

The agricultural production data from 1978 to 2010 are selected in this paper to carry out a research. Specific data are shown in Table I. The amount of chemical fertilizer, the total power of agricultural machinery, crop plantings, the affected area and purchasing price index of agricultural products are selected as the inputs of neural network. The corresponding total agricultural output value is regarded as the network output. In order to eliminate the error caused by the differences between dimension and magnitude of different factors and improve the convergence speed of the network, it is necessary to normalize the original sample data, as shown in the following formula (7).

$$
\bar{x}_{i}=\frac{x_{i}-x_{\min }}{x_{\max }-x_{\min }} \quad i=1,2, \cdots, n
$$

In this formula, $x_{\min }$ and $x_{\max }$ are the minimum and maximum values of the sequence, so that all the data are limited in the interval $[0,1]$. 


\section{B. Model Simulation}

The data from 1978 and 2002, shown in Table I, are regarded as the training samples of neural network, and the corresponding total agricultural output value is regarded as the network output to conduct training for the network and then predict the total agricultural output value from 2003 to 2010. In order to assess the predictions of wavelet neural network, the BP neural network and three-layer wavelet neural network are respectively adopted to train the network, and the network structure of these two neural networks are all $5-8-1$. The gradient-descent training method with momentum is utilized with the training accuracy of 0.001 , and the simulation is carried out under the MatlabR2010 environment. Wavelet neural network reaches the training accuracy after 2869 steps, while the BP neural network reaches the training accuracy after 23562 steps. The predictive results of these two models are shown in Table II.

TABLE I . CHINA TOTAL AGRICULTURAL OUTPUT VALUE AND ITS AFFECTING FACTORS DATA TABLE

\begin{tabular}{|c|c|c|c|c|c|c|}
\hline Year & Fertilizer Input & $\begin{array}{c}\text { Total Power Of } \\
\text { Agricultural Machinery }\end{array}$ & $\begin{array}{l}\text { Sown Area Of } \\
\text { The Crops }\end{array}$ & $\begin{array}{l}\text { Disaster } \\
\text { Area }\end{array}$ & $\begin{array}{c}\text { Index Of Purchasing } \\
\text { Price Of Farm } \\
\text { Product } \\
\end{array}$ & $\begin{array}{l}\text { Total Agricultura } \\
\text { Output Value }\end{array}$ \\
\hline 2010 & 5561.7 & 92780.5 & 160675 & 37426 & 110.9 & 36941.10 \\
\hline 2009 & 5404.4 & 87496.1 & 158614 & 47214 & 97.6 & 30777.50 \\
\hline 2008 & 5239.0 & 82190.4 & 156266 & 39990 & 114.1 & 28044.20 \\
\hline 2007 & 5107.8 & 76589.6 & 153464 & 48992 & 118.5 & 24658.10 \\
\hline 2006 & 4927.7 & 72522.1 & 152149 & 41091 & 101.2 & 21522.28 \\
\hline 2005 & 4766.2 & 68397.8 & 155488 & 38818 & 101.4 & 19613.37 \\
\hline 2004 & 4636.6 & 64027.9 & 153553 & 37106 & 113.1 & 18138.36 \\
\hline 2003 & 4411.6 & 60386.5 & 152415 & 54506 & 104.4 & 14870.10 \\
\hline 2002 & 4339.4 & 57929.9 & 154636 & 46946 & 99.7 & 14931.50 \\
\hline 2001 & 4253.8 & 55172.1 & 155708 & 52215 & 100.3 & 14462.80 \\
\hline 2000 & 4146.4 & 52573.6 & 156300 & 54688 & 96.4 & 13873.60 \\
\hline 1999 & 4124.3 & 48996.1 & 156373 & 49980 & 87.8 & 14106.20 \\
\hline 1998 & 4083.7 & 45207.7 & 155706 & 50145 & 92.0 & 14241.90 \\
\hline 1997 & 3980.7 & 42015.6 & 153969 & 53427 & 95.5 & 13852.50 \\
\hline 1996 & 3827.9 & 38546.9 & 152381 & 46991 & 104.2 & 13539.80 \\
\hline 1995 & 3593.7 & 36118.1 & 149879 & 45824 & 119.9 & 11884.60 \\
\hline 1994 & 3317.9 & 33802.5 & 148241 & 55046 & 139.9 & 9169.20 \\
\hline 1993 & 3151.9 & 31816.6 & 147741 & 48827 & 113.4 & 6605.10 \\
\hline 1992 & 2930.2 & 30308.4 & 149007 & 51332 & 103.4 & 5588.00 \\
\hline 1991 & 2805.1 & 29388.6 & 149586 & 55472 & 98.0 & 5146.40 \\
\hline 1990 & 2590.3 & 28707.7 & 148362 & 38474 & 97.4 & 4954.30 \\
\hline 1989 & 2357.1 & 28067.0 & 146552 & 46991 & 115.0 & 4100.60 \\
\hline 1988 & 2141.5 & 26575.0 & 144866 & 50874 & 123.0 & 3276.88 \\
\hline 1987 & 1999.3 & 24836.0 & 144957 & 42086 & 112.0 & 2837.93 \\
\hline 1986 & 1930.6 & 22950.0 & 144204 & 47135 & 106.4 & 2498.30 \\
\hline 1985 & 1775.8 & 20912.5 & 143626 & 44365 & 108.6 & 2506.40 \\
\hline 1984 & 1739.8 & 19497.2 & 144221 & 31887 & 104.0 & 2181.65 \\
\hline 1983 & 1659.8 & 18022.1 & 143993 & 34713 & 104.4 & 2074.50 \\
\hline 1982 & 1513.4 & 16614.2 & 144757 & 33133 & 102.2 & 1865.30 \\
\hline 1981 & 1406.9 & 15679.8 & 145159 & 39786 & 105.9 & 1635.90 \\
\hline 1980 & 1269.4 & 14745.7 & 146381 & 44526 & 107.1 & 1454.10 \\
\hline 1979 & 1086.3 & 13379.2 & 148476 & 39370 & 122.1 & 1325.30 \\
\hline 1978 & 884.0 & 11749.9 & 150105 & 50790 & 103.9 & 1117.50 \\
\hline
\end{tabular}

TABLE II . AVERAGE OPTIMIZATION RESULTS OF TWO KINDS OF ALGORITHMS

\begin{tabular}{|c|c|c|c|c|c|}
\hline \multirow{2}{*}{ Year } & \multirow{2}{*}{$\begin{array}{c}\text { Actual Total Agricultural } \\
\text { Output Value }\end{array}$} & \multicolumn{2}{|c|}{ Bp Neural Network Model } & \multicolumn{2}{|c|}{ Wavelet Neural Network Model } \\
\hline & & Predictive Value & Relative Error(\%) & Predictive Value & Relative Error(\%) \\
\hline 2003 & 14870.10 & 14468.22 & 2.70 & 14761.98 & 0.73 \\
\hline 2004 & 18138.36 & 17584.59 & 3.05 & 18576.24 & 2.41 \\
\hline 2005 & 19613.37 & 20785.03 & 5.97 & 19394.98 & 1.11 \\
\hline 2006 & 21522.28 & 20294.75 & 5.70 & 21558.02 & 0.17 \\
\hline 2007 & 24658.10 & 24063.14 & 2.41 & 24551.34 & 0.43 \\
\hline 2008 & 28044.20 & 29252.14 & 4.31 & 27648.77 & 1.41 \\
\hline 2009 & 30777.50 & 29273.59 & 4.89 & 30278.61 & 1.62 \\
\hline 2010 & 36941.10 & 34988.41 & 5.29 & 36074.96 & 2.34 \\
\hline
\end{tabular}

BP network is adopted to predict China's total value of agricultural production from 2002 to 2010 and its average relative error is $4.29 \%$. While wavelet neural network is used to carry on the prediction, the average relative error of wavelet neural network is $1.28 \%$. Therefore, the prediction model based on wavelet neural network is superior to the traditional BP neural network model both in the convergence speed and prediction accuracy.

\section{CONCLUSION}

Wavelet neural network is a kind of new network model which is constituted by the combination of the wavelet analysis theory and neural network theory. The wavelet transform has a good time-frequency localization property, while the neural network has self-learning function and good fault tolerance capability, so their combination has a powerful advantage. In this paper, the wavelet neural 
network model is adopted to forecast China's total value of agricultural production, and predictive results show that this model owns a faster convergence speed and higher prediction accuracy compared with traditional BP neural network model.

\section{REFERENCES}

[1] Z. H. Du, X. H. Yu. "Forecasting Method for Farming Gross Output Based on Rough Set and RBF Neural Network," Journal of Luoyang Institute of Science and Technology, vol. 20, pp. 76-79, March 2010

[2] M. Huang, B. T. Cui, S. S. Gu. "Elevator Traffic Flow Prediction Based on Wavelet Neural Networks," Control and Decision, vol. 21, pp. 589-592, May 2006.
[3] X. Z. Fan. "Application of Wavelet Transform in Signal Denoise," Journal of Wuhan University of Science and Technology ( Natural Science Edition ), vol. 27, pp. 286-288, September 2004.

[4] D. H. Zhang, Y. Q. Bi, G. B. Zou, S. F. Jiang. "Overview of Wavelet Neural Network and Its Application in Power Load Forecasting," Proceedings of Electric Power System and Automation, vol. 8, pp. 11-15, August 2004.

[5] N. Li, H. L. Lei, J. D. Han, X. H. Zhu. "Fault Forecast Model of SRG Based on Wavelet Neural Network," Computer Measurement and Control, vol. 19, pp. 1835-1840, August 2011.

[6] X. D. Zhang, J. N. Yu, L. P. Guo, J. G. Zhang, H. R. Fu. "Forecast of Shenzheng Composition Index based on Wavelet Neural Network Model," Journal of Chongqing University of Technology ( Natural Science Edition ), vol. 25, pp. 49-52, June 2011. 